\title{
Precision biomarkers for mood disorders based on brain imaging
}

\section{Identification of biomarkers could facilitate earlier diagnosis and better treatment, say Runsen Chen and colleagues}

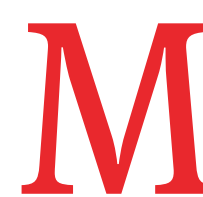
ood disorders are a global public health problem because of their high prevalence, chronicity, and recurrence throughout the lifespan as well as increased risk of mortality. ${ }^{1-3}$ They also impose a heavy economic burden on society because of lost work productivity (occupational disability) and increased use of health services. ${ }^{4}$ Early diagnosis and effective treatment are therefore essential.

Mood disorders are characterised by a significant change in a person's state of mood and include two main subtypes: depressive disorders (major depressive episode and dysthymia) and bipolar disorders (hypomania, mania, or cyclothymia-that is, cycling between depressed and manic states). In 2015, over 300 million people were living with major depressive disorder worldwide, representing $4.4 \%$ of the global population. ${ }^{5}$ A world mental health survey reported that the lifetime and 12 month prevalences of bipolar disorders in the general population were $2.4 \%$ and $1.5 \%$, respectively. ${ }^{6}$

Mood disorders are associated with a widespread cognitive dysfunction, involving higher-order executive function, ${ }^{7}$ reward processing, ${ }^{8}$ and emotional regulation (fig 1 , box 1 ). ${ }^{10}$ These deficits

\section{KEY MESSAGES}

- Mood disorders are among the leading causes of disability across all age groups

- High quality brain imaging data is providing a better understanding of brain mechanisms underlying shared symptoms, heterogeneity, and atypical development of mood disorders

- Such data could allow development of precision biomarkers for mood disorders

- The benefits of a cognitive neuropsychological model could be harnessed to predict treatment outcomes are associated with abnormalities in both the structure and function of specific brain circuits. Interest is growing in developing precision biomarkers for mood disorders based on a deeper understanding of their biological bases by integrating multilevels of data, such as brain imaging, clinical symptoms, and cognitive behaviours. ${ }^{1112}$

Structural and functional magnetic resonance imaging (MRI) can detect subtle deficits in brain structure and function. For example, structural MRI data from more than 20 cohorts worldwide showed that, compared with healthy controls, patients with major depressive disorder had thinner cortical grey matter in the orbitofrontal cortex, cingulate cortex, and insula, ${ }^{13}$ while patients with bipolar disorder had thinner cortical grey matter in the left pars opercularis, left fusiform gyrus, and left rostral middle frontal cortex (fig 1). ${ }^{14}$ Using resting state functional MRI (fMRI), Satterthwaite and colleagues found that the severity of major depressive disorder was associated with diminished connectivity between the amygdala and frontal areas, including both the dorsolateral prefrontal cortices and anterior cingulate cortex. ${ }^{15}$ Studies using task based fMRI showed that patients with mood disorders exhibited atypical neural responses to emotional processing in the medial prefrontal cortex, amygdala, and insula, as well as decreased neural responses to emotional regulation in the dorsolateral prefrontal cortex. ${ }^{16}$ Multimodal brain imaging therefore offers the potential to identify biomarkers for mood disorders that could improve diagnosis and treatment.

\section{Diagnostic confusion}

Current diagnostic systems, including the Diagnostic and Statistical Manual of Mental Disorder and International Classification of Diseases, were developed based on clinical symptoms and signs. The acquired diagnostic categories do not align with the underlying psychopathology or predict treatment response. The diagnostic categories of major depressive disorder and bipolar disorder, for example, share many clinical symptoms, while patients with these diagnoses present with heterogeneous symptoms. ${ }^{17}$

Clinical symptoms may be shared across mood disorders or with psychiatric disorders. For example, major depressive disorder is commonly associated with anxiety symptoms, but these also occur in bipolar disorder and schizophrenia. Recent studies have used brain imaging and transdiagnostic approaches to characterise the neural basis of the shared symptoms. ${ }^{17-19}$ Resting state fMRI data showed that patients with major depressive disorder, bipolar disorder, and schizophrenia had common brain functional deficits. ${ }^{18}$ More generally, recent studies proposed a general psychopathology factor ( $p$ factor) to describe a shared vulnerability to a broad range of symptoms across mental disorders, ${ }^{20}$ and a higher $p$ factor was associated with diminished activation of the frontal pole, anterior cingulate cortex, and anterior insula during executive tasks. ${ }^{19}$ The imbalance in these brain circuits is believed to confer vulnerability to mood disorders as well as other mental disorders, which could be the underlying mechanism of the shared symptoms. Other specific brain circuits related to factors such as anhedonia may therefore give rise to specific diagnoses such as major depressive disorder.

Heterogeneity is also a problem in the current diagnostic system. Mood disorders are increasingly viewed not as a unitary disease but as a heterogeneous clinical syndrome that encompasses multiple subtypes with distinct pathophysiological deficits. Interest is therefore growing in parsing the neurobiological heterogeneity of mood disorders. ${ }^{17}$ Drysdale and colleagues subdivided major depressive disorder into four discrete biotypes using a clustering approach, each type defined by distinct patterns of dysfunctional connectivity in the limbic and frontostriatal systems. ${ }^{21}$ Rather than dividing mood disorders into discrete categories, dimensional approaches are able to parse them into several dimensions using brain imaging, with each dimension representing loadings onto symptoms. ${ }^{17}$ 


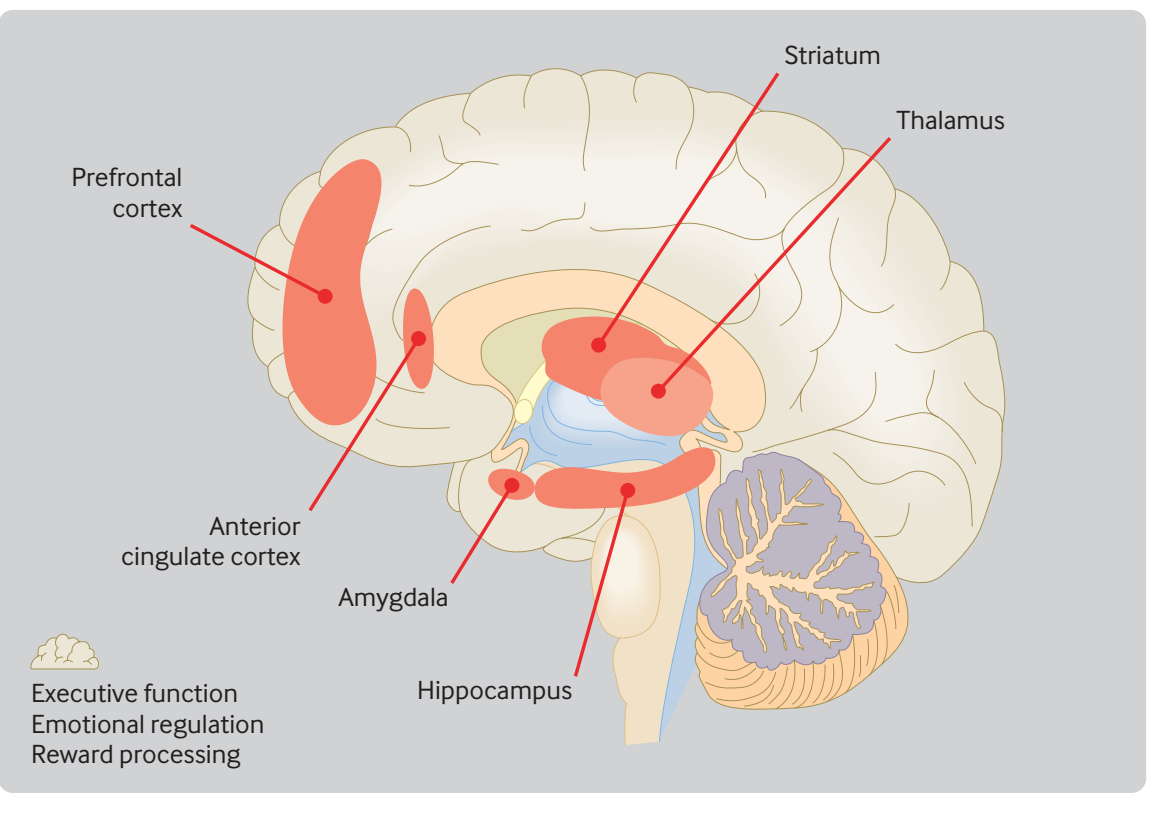

Fig 1 | Brain regions underlying executive function, emotional regulation, and reward processing. The figure was made with reference from Wang and Olson ${ }^{9}$
Dimensional approaches can account for the continuous spectrum from health to disease by including people at risk of illness and considering diagnosable cases as an extreme phenotype. Consistent with these efforts, the US National Institute of Mental Health research domain criteria ${ }^{1112}$ framework invited the scientific and clinical communities to integrate brain imaging, cognition, and clinical symptoms to develop a novel biologically informed nosology and precision biomarkers for mood disorders.

\section{High quality imaging data for early identification}

Precision biomarkers need to be detected at an early stage in the progression of mood disorders to facilitate early intervention.
Accurate identification of the time of onset of a disorder is essential for achieving this goal. Clinical symptoms of mood disorders usually begin in youth, with the mean age of onset being 14.9 years for major depressive disorder ${ }^{22}$ and 20.2 years for bipolar disorders. ${ }^{23}$ Therefore, youth-defined as ranging from childhood to early adulthood-is a critical period for diagnosis and implementation of suitable interventions.

Youth is also a period of considerable brain development, and atypical brain developmental trajectories are related to clinical symptoms and cognitive dysfunction in patients with mood disorders. ${ }^{26}$ For example, longitudinal high resolution MRI showed that relative to healthy youths, those with bipolar

\section{Box 1: Key terms}

- Higher order executive dysfunction-Includes difficulties in planning, organisation, and inhibiting distracting thoughts or behaviours.

- Reward processing dysfunction-Reduced ability to learn or modulate behaviours as a function of reinforcement

- Emotional dysregulation-Includes difficulties in down regulating negative emotions and excessive emotional reactivity

- Precision biomarkers-A system of diagnosis and treatment evaluation for mood disorders based on an understanding of their biological basis

- General psychopathology factor (p factor)-A factor that describes a shared vulnerability to a broad range of symptoms across mental disorders

- Dense fMRI scanning-Obtaining large quantities of functional MRI data in a single person, such as through many hours of scanning

- Cognitive neuropsychological model-In the early stage of antidepressant treatments the primary effect of antidepressants is to modify negative biases in the processing of emotional information disorder had a greater grey matter volume contraction and less white matter expansion in the prefrontal cortex during development. ${ }^{24}$ Xia and colleagues found that mood symptoms were related to dysconnectivity within the frontoparietal system and the pattern of dysconnectivity was strengthened from childhood to adulthood. ${ }^{25}$ Based on these findings, scientists increasingly conceptualise mood disorders as neurodevelopmental disorders. ${ }^{26}$ An understanding of the atypical brain development in patients with mood disorders could allow identification of a biologically informed time of onset for mood disorders, which might be earlier than the onset of clinical symptoms.

Dense sampling shows particular promise for precise characterisation of the atypical development of brain functional organisation in patients with mood disorders. Typical fMRI acquisitions last 5-10 minutes, and may be adequate for characterising group average functional organisation. However, such short acquisitions have limited reliability for describing an individual's brain functional organisation in detail. ${ }^{27} 28$ Recent studies have shown individual variations in the topography of brain organisation that are obscured in group level organisation and can only be reliably detected using dense fMRI scanning data. ${ }^{27} 290$ Dense fMRI protocols acquire a much longer time series of fMRI data on one person, which can be acquired by repeated scanning in multiple sessions. Such protocols reveal specific topography details for individuals that are both reliable and reproducible and could be useful in identifying precision biomarkers for mood disorders. ${ }^{31}$

Recently, Cui and colleagues used 27 minute fMRI acquisitions, which is much longer than traditional acquisitions, to show that the topography of brain functional organisation is refined during youth. ${ }^{32}$ Such characterisation of typical brain development in youth is a prerequisite for understanding differences in brain development in mood disorders. Results also showed that the individual variation in brain functional organisation predicted executive function, ${ }^{32}$ which is typically impaired in mood disorders. These findings suggest that the topography of functional organisation revealed by dense fMRI scanning could be helpful in understanding the atypical neurodevelopmental trajectories of mood disorders. However, dense fMRI scanning data are still lacking for healthy youths as well as for youths with mood disorders, 
and the neuroscience and psychiatry communities should concentrate on this effort.

As well as dense fMRI scanning data on individuals, data from large samples are also essential to characterise how mood disorders differ from the typical brain developmental trajectory. The research community has publicly released several youth datasets with large sample sizes, such as the Philadelphia neurodevelopmental cohort (box 2). ${ }^{33}$ The Philadelphia cohort includes young people representing a spectrum from a healthy to a diseased state and is therefore a great resource for identification of the time of onset of mood disorders. Future efforts should collect datasets with both a large sample size and dense fMRI scanning to better characterise the time of onset.

\section{Early biomarkers of treatment outcomes}

Identifying early biomarkers of treatment outcomes is vital for evaluating treatment strategies for mood disorders. A recent theory of antidepressant drug action-the cognitive neuropsychological model-suggests that drugs do not act primarily on mood but rather modify biases in the cognitive processing of emotional information in the brain (fig 2). ${ }^{3435}$ fMRI has been used to study changes in the brain associated with positive or negative biases in emotional processing that occur when patients with mood disorders and healthy volunteers take antidepressants. For instance, Godlewska and colleagues ${ }^{36}$ found that, compared with placebo, treatment with the antidepressant escitalopram for 7 days normalised neural responses in patients with major depressive disorder by reducing the response of the amygdala to negative stimuli (fearful facial expressions). Importantly, the neural changes seen during the early stages of antidepressant treatment were independent of subjective mood changes, suggesting that antidepressant drugs act directly on neural functions that are relevant to major depressive

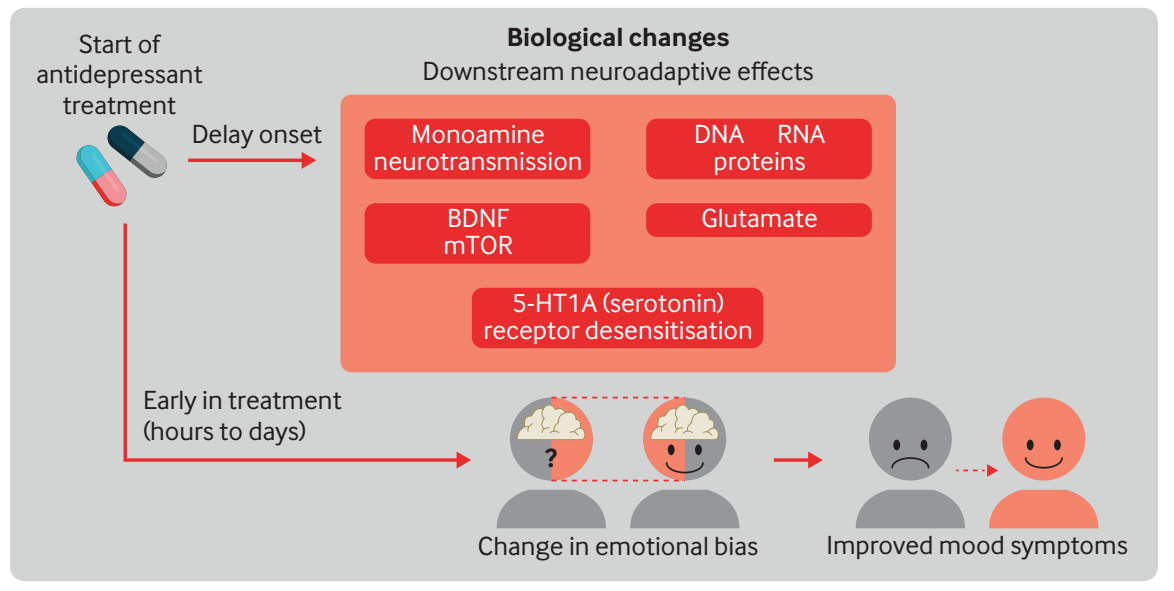

Fig 2 | Antidepressants produce positive biases in emotional processing from the start of treatment. This is an automatic effect which does not affect subjective mood state immediately (BDNF=brain derived neurotropic factor, $\mathrm{mTOR}=$ mammalian target of rapamycin) disorder before producing any effects on mood.

Interestingly, studies have consistently shown that antidepressant induced early changes in neural response to emotional processing tasks predict treatment outcomes. ${ }^{37}$ A recent study assessed changes in neural response to emotional facial expressions before and after 7 days of treatment with escitalopram in 35 patients with major depressive disorder who had not previously had drug treatment. ${ }^{38}$ Based on the criterion of $50 \%$ reduction in depressive symptoms at the end of six weeks' treatment, 22 patients were classified as responders to the treatment and 13 as non-responders. Compared with the non-responders, responders showed decreased neural activity to fearful versus happy faces in the amygdala, insula, anterior and posterior cingulate cortices, bilateral supramarginal gyri, and thalamus. These results suggest that early changes in neural responses to fearful faces are predictive of clinical response to treatment for major depressive disorder.

Studies of neural responses could therefore be useful in testing the efficacy

\section{Box 2: Open access brain developmental datasets in youth}

- Philadelphia neurodevelopmental cohort: https://www.ncbi.nlm.nih.gov/projects/gap/cgibin/study.cgi?study_id=phs000607.v3.p2

- Adolescent Brain Cognitive Development: https://abcdstudy.org

- Human Connectome Project Development: https://humanconnectome.org/study/hcplifespan-development

- Pediatric Imaging, Neurocognition, and Genetics (PING) Data Repository: http://pingstudy. ucsd.edu/

- Healthy Brain Network Biobank: https://data.healthybrainnetwork.org/main.php

- IMAGEN: http://www.imagen-europe.com/ of novel drugs in both patients and healthy volunteers. Neural responses could also serve as an early biomarker of treatment efficacy and improve understanding of mechanisms of action, including those underlying drugs targeting N-methyl-Daspartate receptors (such as ketamine ${ }^{37}$ ). Candidate drugs are typically screened using animal models, which have low predictive validity, and a lack of efficacy in translation to humans is the main reason for failure in drug development. ${ }^{39}$ Ensuring that new drugs affect the core measures of emotional or cognitive function during the early phase of drug development could reduce costs of failed clinical trials. A better understanding of the cognitive neuropsychological effects of psychopharmacological drugs and using neural assessment to predict treatment response may in future allow better patient stratification for mood disorder treatment.

\section{Conclusion}

Development and validation of precision biomarkers for mood disorders is urgently needed for early diagnosis and treatment evaluation. However, the overlap and heterogeneity in mood disorders impede this progress. Brain imaging, which can detect brain structural and functional changes, is one promising way to solve this problem and identify biomarkers that can cut across different facets of disorders of mood and emotion. This will require a combination of brain imaging, cognitive neuroscience, experimental modelling, and computational techniques (box 3 ). We encourage the close collaborations between a diverse research community for this endeavour. 


\section{Box 3: Future directions in precision biomarkers for mood disorders}

- Understand the neural substrate underlying shared symptoms using transdiagnostic approach, which cut across the diagnostical categories

- Identify biologically informed biotypes of mood disorder and their distinct brain deficits

- Characterise how the brain development of patients with mood disorders deviates from the typical trajectory in youth

- Use brain imaging data to predict the emergence of mood disorders and treatment outcome

- Advance the cognitive neuropsychological model with improved experimental designs to better evaluate the treatment outcomes of psychopharmacological therapies

We thank Ying Wang for help with the figures.

Contributors and sources: GW is the president of Beijing Anding Hospital, Capital Medical University, and the director of the National Clinical Research Center for Mental Disorders, China. $\mathrm{RC}$ is a mental health researcher, specialising in psychopharmacology, emotion, and clinical psychology. ZC is a postdoctoral fellow investigating typical and atypical brain development using brain imaging and computational techniques. $\mathrm{LC}$ is a researcher within the experimental medicine and adult mental health themes, part of the Oxford Health Biomedical Researcher Centre. TS is director of the lifespan informatics and neuroimaging centre at the University of Pennsylvania Perelman School of Medicine. $\mathrm{CH}$ is the director of the psychopharmacology and emotional research laboratory at the University of Oxford. GW is a guarantor of the article. RC and ZC drafted the article. All authors contributed to critical revision of the article and approved the final manuscript. RC and ZC contributed equally to this article and share first authorship.

Competing interests: We have read and understood the BMJ policy on declaration of interests and have the following interests to declare: GW declares funding from the National Key Research and Development Program of China (2016YFC1307200; 2017YFA0505700), the Capital's Funds for Health Improvement and Research (2018-1-2121), and the Capital's Science and Technology Talent Project (Z181100006318009). LP and CH are supported by the NIHR Oxford Health Biomedical Research Centre. The views expressed are those of the authors and not necessarily those of the NHS, the NIHR, or the Department of Health. $\mathrm{CH}$ receives consultancy fees from p1vital, Lundbeck, Sage Therapeutics, and I\&J. The funders had no role in the design or conduct of the study, collection, management, analysis, or the interpretation of the data.

Provenance and peer review: Commissioned; externally peer reviewed.

This article is part of a series launched at the Chinese Stroke Association annual conference on 10 October 2020, Beijing, China. Open access fees were funded by the National Science and Technology Major Project. The BM/ peer reviewed, edited, and made the decision to publish these articles.

Runsen Chen, researcher ${ }^{1,2}$

Zaixu Cui, postdoctoral fellow ${ }^{3}$

Liliana Capitão, research coordinator ${ }^{2,4}$

Gang Wang, professor ${ }^{1}$

Theodore D Satterthwaite, associate professor ${ }^{3}$

Catherine Harmer, professor of cognitive

neuroscience ${ }^{2,4}$

${ }^{1}$ National Clinical Research Center for Mental

Disorders, Beijing Key Laboratory of Mental Disorders,

Beijing Anding Hospital, and Advanced Innovation

Center for Human Brain Protection, Capital Medical

University, Beijing, China
${ }^{2}$ Department of Psychiatry, University of Oxford, Oxford, UK

${ }^{3}$ Department of Psychiatry, University of Pennsylvania, Philadelphia, PA 19104, USA

${ }^{4}$ Oxford Health NHS Foundation Trust, Warneford Hospital, Oxford, UK

Correspondence to: G Wang

gangwangdoc@ccmu.edu.cn

\section{(?) (1) O OPEN ACCESS}

This is an Open Access article distributed in accordance with the Creative Commons Attribution Non Commercial (CC BY-NC 4.0) license, which permits others to distribute, remix, adapt, build upon this work non-commercially, and license their derivative works on different terms, provided the original work is properly cited and the use is non-commercial. See: http://creativecommons.org/ licenses/by-nc/4.0/.

\section{Check for updates}

1 Vieta E, Berk M, Schulze TG, et al. Bipolar disorders Nat Rev Dis Primers 2018;4:18008. doi:10.1038/ nrdp. 2018.8

2 Belmaker RH, Agam G. Major depressive disorder. N Engl J Med 2008;358:55-68. doi:10.1056/ NEJMra073096

3 Carvalho AF, Firth J, Vieta E. Bipolar disorder. N Engl J Med 2020;383:58-66. doi:10.1056/ NEJMra1906193

4 Simon GE. Social and economic burden of mood disorders. Biol Psychiatry 2003;54:208-15. doi:10.1016/S0006-3223(03)00420-7

5 World Health Organization. Depression and other common mental disorders: global health estimates. World Health Organization; 2017. https://apps.who. int/iris/bitstream/handle/10665/254610/WHOMSD-MER-2017.2-eng.pdf

6 Merikangas KR, Jin R, He J-P, et al. Prevalence and correlates of bipolar spectrum disorder in the world mental health survey initiative. Arch Gen Psychiatry 2011;68:241-51. doi:10.1001/ archgenpsychiatry.2011.12

7 Cotrena C, Branco LD, Shansis FM, Fonseca RP. Executive function impairments in depression and bipolar disorder: association with functional impairment and quality of life. J Affect Disord 2016;190:744-53. doi:10.1016/j. jad.2015.11.007

8 Panchal P, Kaltenboeck A, Harmer CJ. Cognitive emotional processing across mood disorders. CNS Spectr 2019;24:54-63. doi:10.1017/ S109285291800130X

9 Wang Y, Olson IR. The original social network: white matter and social cognition. Trends Cogn Sci 2018;22:504-16. doi:10.1016/j. tics.2018.03.005

10 Leppänen JM. Emotional information processing in mood disorders: a review of behavioral and neuroimaging findings. Curr Opin
Psychiatry 2006;19:34-9. doi:10.1097/01. yco.0000191500.46411.00

11 Insel TR. The NIMH research domain criteria (RDoC) project: precision medicine for psychiatry. Am J Psychiatry 2014;171:395-7. doi:10.1176/appi. ajp. 2014.14020138

12 Insel T, Cuthbert B, Garvey M, et al. Research domain criteria (RDoC): toward a new classification framework for research on mental disorders. Am J Psychiatry 2010;167:748-51. doi:10.1176/appi. ajp.2010.09091379

13 Schmaal L, Hibar DP, Sämann PG, et al. Cortical abnormalities in adults and adolescents with major depression based on brain scans from 20 cohorts worldwide in the ENIGMA Major Depressive Disorder Working Group. Mol Psychiatry 2017;22:900-9. doi: $10.1038 / \mathrm{mp} .2016 .60$

14 Hibar DP, Westlye LT, Doan NT, et al. Cortical abnormalities in bipolar disorder: an MRI analysis of 6503 individuals from the ENIGMA bipolar disorder working group. Mol Psychiatry 2018;23:932-42. doi:10.1038/mp.2017.73

15 Satterthwaite TD, Cook PA, Bruce SE, et al. Dimensional depression severity in women with major depression and post-traumatic stress disorde correlates with fronto-amygdalar hypoconnectivty. Mol Psychiatry 2016;21:894-902. doi:10.1038/ mp.2015.149

16 Malhi GS, Byrow Y, Fritz K, et al. Mood disorders: neurocognitive models. Bipolar Disord 2015;17(Suppl 2):3-20. doi:10.1111/ bdi. 12353

17 Kaczkurkin AN, Moore TM, Sotiras A, Xia CH, Shinohara RT, Satterthwaite TD. Approaches to defining common and dissociable neurobiological deficits associated with psychopathology in youth Biol Psychiatry 2020;88:51-62. doi:10.1016/j. biopsych.2019.12.015

18 Ma Q, Tang Y, Wang F, et al. Transdiagnostic dysfunctions in brain modules across patients with schizophrenia, bipolar disorder, and major depressive disorder: a connectome-based study. Schizophr Bull 2020;46:699-712. doi:10.1093/ schbul/sbz111

19 Shanmugan S, Wolf DH, Calkins ME, et al. Common and dissociable mechanisms of executive system dysfunction across psychiatric disorders in youth. Am J Psychiatry 2016;173:517-26. doi:10.1176/appi. ajp.2015.15060725

20 Caspi A, Houts RM, Belsky DW, et al. The p factor: one general psychopathology factor in the structure of psychiatric disorders?Clin Psychol Sci 2014:2:11937. doi:10.1177/2167702613497473

21 Drysdale AT, Grosenick L, Downar J, et al. Resting-state connectivity biomarkers define neurophysiological subtypes of depression. Nat Med 2017;23:28-38. doi:10.1038/nm.4246

22 Lewinsohn PM, Clarke GN, Seeley JR, Rohde P. Major depression in community adolescents: age at onset, episode duration, and time to recurrence. J Am Acad Child Adolesc Psychiatry 1994;33:809-18. doi:10.1097/00004583-199407000-00006

23 Morken G, Vaaler AE, Folden GE, Andreassen OA, Malt UF. Age at onset of first episode and time to treatment in in-patients with bipolar disorder. $\mathrm{Br}$ J Psychiatry 2009;194:559-60. doi:10.1192/bjp. bp. 108.054452

24 Najt P, Wang F, Spencer L, et al. Anterior cortical development during adolescence in bipolar disorder. Biol Psychiatry 2016;79:303-10. doi:10.1016/j. biopsych.2015.03.026

25 Xia CH, Ma Z, Ciric R, et al. Linked dimensions of psychopathology and connectivity in functional brain networks. Nat Commun 2018;9:3003. doi:10.1038/ s41467-018-05317-y

26 Insel TR. Mental disorders in childhood: shifting the focus from behavioral symptoms to neurodevelopmental trajectories. JAMA 2014;311:1727-8. doi:10.1001/ jama.2014.1193 
27 Laumann TO, Gordon EM, Adeyemo B, et al. Functional system and areal organization of a highly sampled individual human brain. Neuron 2015;87:657-70. doi:10.1016/j. neuron.2015.06.037

28 Zuo XN, Xu T, Milham MP. Harnessing reliability for neuroscience research. Nat Hum Behav 2019;3:76871. doi:10.1038/s41562-019-0655-x

29 Gordon EM, Laumann TO, Gilmore AW, et al. Precision functional mapping of individual human brains. Neuron 2017;95:791-807.e7. doi:10.1016/j. neuron. 2017.07.011

30 Braga RM, Buckner RL. Parallel interdigitated distributed networks within the individual estimated by intrinsic functional connectivity. Neuron 2017;95:457-471.e5. doi:10.1016/j. neuron.2017.06.038

31 Gratton C, Kraus BT, Greene DJ, et al. Defining individual-specific functional neuroanatomy for precision psychiatry. Biol Psychiatry 2020;88:28-39. doi:10.1016/j.biopsych.2019.10.026
$32 \mathrm{Cui} \mathrm{Z}$, Li H, Xia CH, et al. Individual variation in functional topography of association networks in youth. Neuron 2020;106:340-353.e8. doi:10.1016/j.neuron.2020.01.029

33 Satterthwaite TD, Elliott MA, Ruparel K, et al. Neuroimaging of the Philadelphia neurodevelopmental cohort. Neuroimage 2014;86:544-53. doi:10.1016/j. neuroimage.2013.07.064

34 Harmer CJ, Goodwin GM, Cowen PJ. Why do antidepressants take so long to work? A cognitive neuropsychological model of antidepressant drug action. Br J Psychiatry 2009;195:102-8. doi:10.1192/bjp.bp.108.051193

35 Zghoul T, Cowen PJ, Harmer Cl. A perspective: from the serotonin hypothesis to cognitive neuropsychological approaches. The serotonin system. Elsevier, 2019: 95-104.

36 Godlewska BR, Norbury R, Selvaraj S, Cowen PJ, Harmer CJ. Short-term SSRI treatment normalises amygdala hyperactivity in depressed patients.
Psychol Med 2012;42:2609-17. doi:10.1017| S0033291712000591

37 Harmer C), Duman RS, Cowen PJ. How do antidepressants work? New perspectives for refining future treatment approaches. Lancet Psychiatry 2017;4:409-18. doi:10.1016/S22150366(17)30015-9

38 Godlewska BR, Browning M, Norbury R, Cowen PJ, Harmer C). Early changes in emotional processing as a marker of clinical response to SSRI treatment in depression. Transl Psychiatry 2016;6:e957. doi:10.1038/tp.2016.130

39 Pringle A, Harmer CJ. The effects of drugs on human models of emotional processing: an account of antidepressant drug treatment. Dialogues Clin Neurosci 2015;17:477-87.

Cite this as: $B M J$ 2020;371:m3618 http://dx.doi.org/10.1136/bmj.m3618 